\title{
Evolutionary game theory-based power control for uplink NOMA
}

\author{
Sidra Riaz, Jihwan Kim and Unsang Park* \\ Department of Computer Science and Engineering, Sogang University \\ 35 Baekbeom-ro, Mapo-gu, South Korea \\ [e-mail: \{sidra, kimjihwan, unsangpark\}@ sogang.ac.kr] \\ *Corresponding author: Unsang Park
}

Received June 29, 2017; revised November 14, 2017; accepted December 23, 2017; published June 30, 2018

\begin{abstract}
Owing to the development of Internet of Things (IoT), the fifth-generation (5G) wireless communication is going to foresee a substantial increase of mobile traffic demand. Energy efficiency and spectral efficiency are the challenges in a $5 \mathrm{G}$ network. Non-orthogonal multiple access (NOMA) is a promising technique to increase the system efficiency by adaptive power control (PC) in a 5G network. This paper proposes an efficient PC scheme based on evolutionary game theory (EGT) model for uplink power-domain NOMA system. The proposed PC scheme allows users to adaptively adjusts their transmit power level in order to improve their payoffs or throughput which results in an increase of the system efficiency. In order to separate the user signals, a successive interference cancellation (SIC) receiver installed at the base station (BS) site. The simulation results demonstrate that the proposed EGT-based PC scheme outperforms the traditional game theory-based PC schemes and orthogonal multiple access (OMA) in terms of energy efficiency and spectral efficiency.
\end{abstract}

Keywords: Non-orthogonal multiple access, power NOMA in 5G, adaptive power control, evolutionary game theory, spectral efficiency, energy efficiency

This work was supported by Basic Science Research Program through the National Research Foundation of Korea (NRF) funded by the Ministry of Education (2014R1A1A2058084). 


\section{Introduction}

Rapid development of Internet of Things (IoT) and mobile Internet has propelled $1000 \%$ data-traffic increase by 2020 [1]. As hand-held devices with low-power consumption and low cost are the need of the hour, there is a dire necessity of a power-efficient wireless system. Moreover, technologies that can mitigate or control inter-cell interferences need to be the focus of research. The fifth-generation $(5 \mathrm{G})$ systems are expected to support very higher data rates, massive connectivity, and energy efficient services [2]. To meet these targets, a non-orthogonal multiple access (NOMA) technique has been widely studied due to its enhanced spectral efficiency. In contrast to the conventional orthogonal multiple access schemes, the NOMA scheme can simultaneously serve multiple users by power domain multiplexing at the transmitter and successive interference cancellation (SIC) at the receiver [2]. Fig. 1, shows the uplink NOMA with SIC receiver. For uplink NOMA, the key issue is that how the base station (BS) can split the composite power signals arriving from multiple users in the power domain. To employ a NOMA system, a specific power control (PC) scheme is required [2]. A game theory (GT) tool is one of the suitable candidates to solve the PC problems [3]. The evolutionary GT (EGT) is similar to traditional GT; however, the former theory focuses on the dynamics of the strategy adaptation in the population involved in the game. Initially, the EGT scheme was developed to explain the evolution of biological populations, it has recently been applied to wireless communications [4-6] showing better payoff and improved system performances. Thus we develop our motivation to use EGT based PC scheme in power domain NOMA systems.

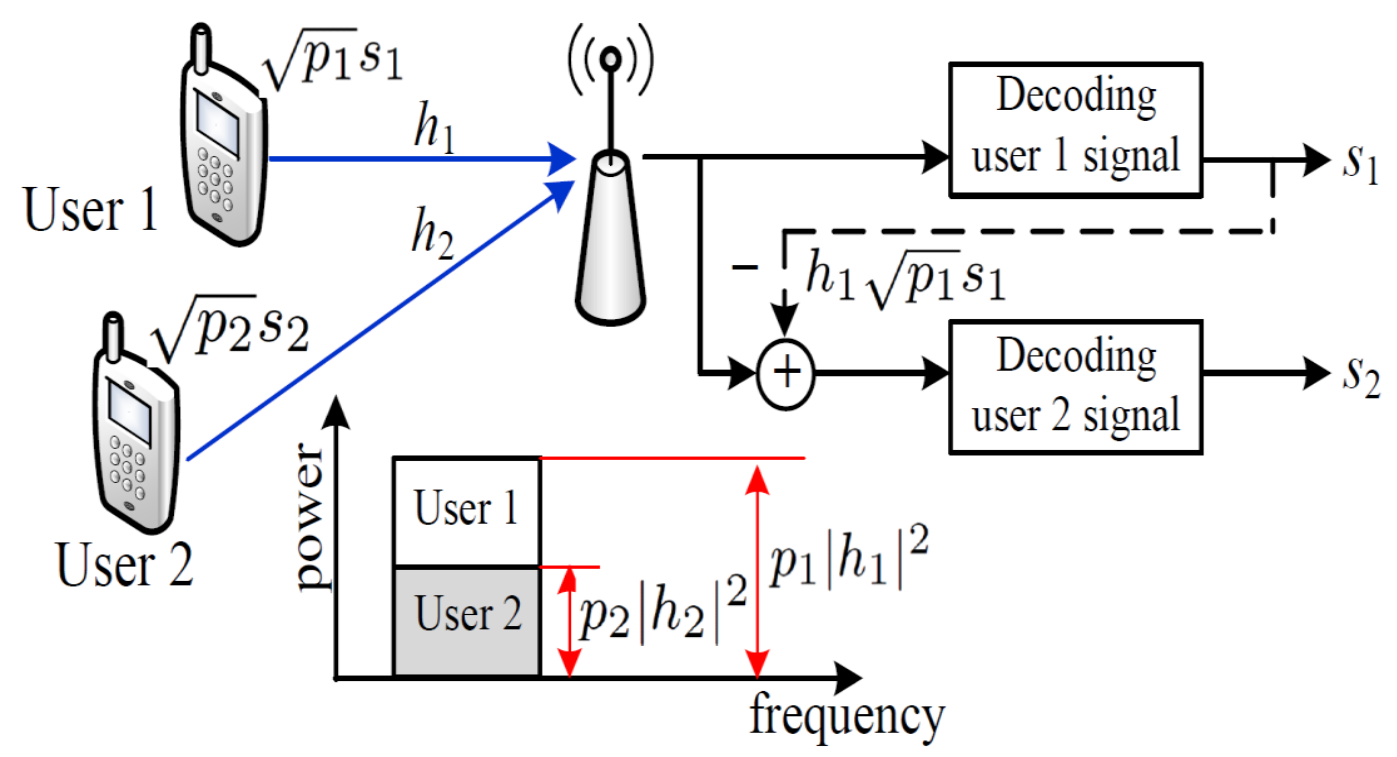

Fig. 1. The SIC receiver with two users sending their signals over a wireless channel in an uplink NOMA network

The related works in uplink NOMA and game theory domain are discussed in the following section. 


\section{Related Work}

Many researchers have developed the GT-based PC models in various wireless communication systems. Ginde et al. [7] proposed a joint link adaptation and PC algorithm for a general packet radio service (GPRS) system, where a non-cooperative GT was applied to solve a multi-rate PC problem. Their presented GT-based PC scheme shows acceptable levels of interference to other co-channel links by adjusting the power and modulation levels. Long et al. [8] proposed a reinforcement learning-based PC algorithm for wireless ad hoc networks with repeated games. The authors presented that the learning dynamics in the repeated game eventually converges to pure Nash equilibrium (NE). Zappone et al. [9] developed a non-cooperative PC strategies for the uplink of relay-assisted DS/CDMA wireless networks by using game theoretic tools and discussed the existence and uniqueness of the NE. Li et al. [10] presented GT in the uplink PC problem of a WiMAX system, where co-channel users are game participants and their uplink power is used as a strategy to achieve NE. Panaousis et al. [11] presented power control scheme in a shared medium by using both cooperative and non-cooperative games. In a non-cooperative power control game, the author found the Nash equilibrium (NE) and in a cooperative game, they assumed a central entity called coordinator which announces the Nash bargain solution (NBS) to the access points (APs). In a CDMA system, an uplink power control scheme was presented by Alpcan et al. [12] by following the concept of non-cooperative game. That study demonstrated that there exists a unique Nash equilibrium (NE) in their framework. Kang et al. [23] presented a PC algorithm for machine type communications (MTC) in a NOMA network. They proposed a cost function which reflects the power consumption and singal-to-intereference-plus-noise ratio (SINR) for each device in a non-cooperative game model. Qian et al. [24] presented a NOMA system with an SIC receiver at the uplink side. A joint optimization of BS association and PC is explored to minimize the power consumption and maximize the system-wide utility. Sung and Fu [25] presented an uplink NOMA system with two cells and compared the NOMA with OMA. Several previous studies presented traditional game theoretic approaches to solve the PC problem in the specific network; relying on several assumptions that are not practical in the uplink NOMA system for 5G networks [7-12]. In contrast to earlier work, this paper explores the effectiveness of adopting an EGT-based PC scheme for uplink NOMA in a $5 \mathrm{G}$ network. We also compare the proposed EGT-based PC approach with the traditional GT-based PC schemes.

\subsection{Technical Contribution}

1. We exploit the use of the GT approaches for uplink power control in a NOMA scheme for the $5 \mathrm{G}$. The SIC receiver is proposed to be on the BS site for user signal separation. EGT based power control scheme allows the users to adjust their power levels adaptively in order to improve their own payoff (utility function) while achieving the satisfaction of other users through an evolutionary process.

2. Simulations are carried out in order to show the improved network performance under an evolutionary power controlled NOMA network.

The remainder of this paper is organized as follows. In Section 2, the overview of NOMA system is provided and our proposed EGT-based power control scheme for uplink NOMA is explained. In Section 3, we present our simulation results in comparison with state-of-the-art PC schemes. Section 4 draws conclusions and outlines directions of the further research. 


\section{Proposed Method}

We consider a two cell uplink NOMA system, where $K$ users simultaneously share the same uplink channel. Let $h_{k}$ denote the channel coefficient for the $k^{\text {th }}$ user. Then, the received signal at the BS can be expressed as follows (see Eq. (1)):

$$
Y=\sum_{k=1}^{K} h_{k} \sqrt{p_{k}} s_{k}+w
$$

where $p_{k}$ and $S_{k}$ are the transmit power and the transmit message signal from the $k^{\text {th }}$ user, respectively. The variable $w$ represents the additive white Gaussian noise (AWGN) with zero mean and $\sigma^{2}$ variance. For the simplicity of the practical implementation, the transmit power of users is assumed to be classified into $N=6$ discrete levels. In order to split the diverse arrived power from users, an SIC receiver is employed at the BS which decodes the $k^{\text {th }}$ user signals considering the other signals as Gaussian interference. Fig. 1 shows an SIC receiver in an uplink NOMA system with two users.

\subsection{EGT-based Power Control for Uplink NOMA}

An EGT model consists of players, population, strategy, and payoff, i.e., $G=\left\{K, \Omega, \nabla,\left\{u_{k}\right\}\right\}$, where $K$ is the number of simultaneous players (wireless system users) on a single channel, $\Omega$ is the population which refers to the set of players, $\nabla$ is the non-empty set of the strategies, and $u_{k}$ is the utility function of player $k$. In the uplink NOMA system, each user; who is a player of the game chooses a transmit power level in the range [ $\left.p_{\min }, p_{\max }\right]$, where $p_{\min }$ and $p_{\max }$ are the pre-defined minimum and maximum power levels, respectively. Each player selects a single strategy from $\nabla$ in order to maximize its expected payoff, where the payoff is the utility function in a game theory model. The set of PC strategies expressed as follows (see Eq. (2)):

$$
\nabla=\left\{\prod_{n=1}^{N=6} p_{n} \mid p_{n} \in\left[p_{\min }, p_{\max }\right\}\right.
$$

where $p_{n}$ is the selected power level of a user.

In our proposed game model, the utility function for player $k$ is defined as throughput per transmit power of player $k$ (see Eq. (3)).

$$
u_{k}=\frac{R_{k}}{p_{n}(k)}
$$

where $p_{n}(k)$ is the transmit power level for user $k$ and $R_{k}$ is the throughput of user $k$. In the uplink NOMA system, the SIC receiver decodes the users' signals in the descending order of the received power level. That is, the SIC receiver first decodes the strongest user's signal with interference and then decodes the next strongest user's signal after SIC using the decoded signals. To simplify the notation, we define the effective received power of user $k$ as $\alpha_{k}=p_{k}\left|h_{k}\right|^{2}$. Let $k^{\prime}$ be the ordered index of user $k$ in the set of users listed in the descending 
order of their effective received power, $\alpha_{k}$. We define an order mapping function as $\phi\left(k^{\prime}\right)=k$. The effective received power at the BS listed in the descending order as shown in Eq. (4):

$$
\alpha_{\phi(1)}>\alpha_{\phi(2)}>\cdots>\alpha_{\phi(K)}
$$

Assuming perfect SIC, the data rate of a player $k$ can be expressed as shown in Eq. (5).

$$
R_{k}=B \log _{2}\left(1+\frac{\alpha_{k}}{\sum_{i=k^{\prime}+1}^{K} \alpha_{\phi(i)}+\sigma^{2}}\right)
$$

where $\mathrm{B}$ is the bandwidth and $k^{\prime}=\phi^{-1}(k)$. The sum rate of the NOMA system is given by Eq. (6).

$$
R=\sum_{k=1}^{K} R_{k}
$$

In the EGT, a player reproduces (i.e., replicates) itself through the process of mutation and selection. A player with a higher payoff can replicate itself faster. The reproduction process takes place over time and therefore, can be modeled by using a set of ordinary differential equations called replicator dynamics (RD) [5]. Let $x_{n}$ be the selection probability that a player chooses the $n^{\text {th }}$ power level, where there are $N=6$ possible power levels (i.e., there are $N$ strategies for players). If the payoff of $n^{\text {th }}$ strategy is high as compared to the other strategies, the selection probability for $n^{\text {th }}$ strategy increases proportionally to the expected payoff. The selection probabilities of population, $\boldsymbol{X}=\left(\begin{array}{cccc}x_{11} & x_{12} & \cdots & x_{n 1} \\ x_{12} & x_{22} & \cdots & x_{n 2} \\ \vdots & \vdots & \ddots & \vdots \\ x_{1 \Omega} & x_{2 \Omega} & \cdots & x_{n \Omega}\end{array}\right)$ can be denoted by $\Omega=50$ selection probability vectors where $x_{11}$ represents selection probability of user 1 with selected power level $n=1$. Let $\dot{x}_{n}$ represent the variation of $x_{n}$. The RD can be defined as follows (see Eq. (7)):

$$
\dot{x}_{n}=\left(\lambda\left(x_{n}(t)\right) \times x_{n}\right) \times(\pi(t)-\bar{\pi}(t))
$$

where $\lambda\left(x_{n}(t)\right)$ is a risk control factor that can control the speed of convergence, $\pi(t)$ is the payoff of the individual user, and $\bar{\pi}(t)$ is the average payoff of the entire population $(\Omega=50)$. The values of $\pi(t)$ and $\bar{\pi}(t)$ are calculated using Eq. (8).

$$
\pi(t)=\sum_{n, m=1}^{N} x_{n} \cdot J(n, m), \quad \bar{\pi}(t)=\sum_{n=1}^{N} x_{n} \cdot J(n, \boldsymbol{X})
$$

where $J(n, m)$ is the expected payoff for a player using $n^{\text {th }}$ strategy when it encounters a player with $m^{\text {th }}$ strategy and $J(n, X)$ is the payoff a user with selected strategy $n$ and encounters with rest of the players with a state set $X$. In Eq. (7) the control factor of $\lambda\left(x_{n}\right)$ can be regarded as a 
risk control function. By using the expected utility theory, all players can be classified as either risk-averse or risk-seeking players, where risk-averse players do not necessarily maximize their utility in comparison with risk-seeking players. For player $k$ with the transmit power of $p_{n}$, if $\arg \max _{k} u_{k}\left(p_{n} \mid p_{n}=n^{\text {th }}\right.$ power level $\left.)=\arg \max _{k} \dot{x}_{k}\right)$, the player is regarded as a risk-averse player. By using a constant absolute risk utility function of the expected utility theory, the control factor of $\lambda\left(x_{n}\right)$ is defined as follows (see Eq. (9)) [13]:

$$
\lambda\left(x_{n}\right)=\frac{1}{\beta}\left(e^{\beta x_{n}}-1\right)
$$

where if the player is a risk-averse player, we set $\beta=-1$; otherwise, we set $\beta=1$.

The iterative EGT-based power allocation is summarized in Algorithm 1. At the initial iteration $\left(t_{0}\right)$ the strategy selection probabilities are equally set as: $x_{n}\left(t_{0}\right)=\frac{1}{N}$. Each user repeatedly updates its power level in order to obtain a higher payoff. If the change of RD is within a pre-defined value, e.g., $\varepsilon=0.005$, and no players change their current strategies, the iteration stops and all users have reached their equilibrium stable strategies (ESS).

\section{Algorithm 1. Iterative EGT-based power allocation for uplink NOMA}

1: Set $x_{n}(t)=\frac{1}{N}$ at initial time $\left(t_{0}\right)$, where $N$ is the number of discrete power levels available for users.

\section{2: START}

3: $\quad t \leftarrow t+1$

4: Each user selectes a power level according to the strategy selection probability, $x_{n}$

5: Each user is classified as either a risk-averse player or a risk-seeking player, and the control factor of $\lambda(\cdot)$ is determined from Eq. (9).

6: The strategy selection probabilities are updated as follows:

$$
\dot{x}_{n}=\lambda\left(x_{n}(t)\right) \cdot x_{n}(t)\left(\pi_{n}(t)-\bar{\pi}(t)\right)
$$

7: if $\left.\left|x_{n}(t)-x_{n}(t-1)\right| \leq \varepsilon\right)$ i.e., no player change its current strategy

8: $\quad$ Exit because the system has reached ESS

9: else

10: $\quad$ Update strategies

11: $\quad \dot{x}_{n}=\lambda\left(x_{n}(t)\right) \cdot x_{n}(t)\left(\pi_{n}(t)-\bar{\pi}(t)\right)$

12: end

13: END 


\section{Simulation Results and Analysis}

We evaluated the performance of the proposed EGT-based PC scheme under an uplink NOMA system in comparison with the approaches presented by Long et al. [8], Ginde et al. [7], Kang et al. [23], and Sung et al. [25]. These schemes ([7-8], [23, 25]) were developed under different wireless network scenarios using different simulation parameters. For the simplicity however, we used the same simulation parameters for evaluating all the previous schemes with our proposed EGT-based PC algorithm.

For our implementation of the NOMA system, we assumed the number of users that simultaneously transmit at each subcarrier are limited to two. The subcarrier allocation is done by Global Objective Maximization (GOM) [19], which allocates a subcarrier to the user that achieves the maximum increase in the throughput. Each user generates a fixed sized packet with a Poisson arrival distribution [14] and the service request is $285 \mathrm{Kbps}$. Users are uniformly distributed [15] in a cell with a radius of 389 meters and are selected according to the maximum rate scheduling [16]. The transmit power of each user is [15 dBm, $23 \mathrm{dBm}]$ with $N=6$ discrete levels. The total network bandwidth capacity is $15 \mathrm{Mbps}$ and the noise power is $-173 \mathrm{dBm} / \mathrm{Hz}$. The radio channel follows a lognormal shadowing distribution with a mean of 0 $\mathrm{dB}$ and variance of $8 \mathrm{~dB}$ [8]. All the simulation parameters are summarized in Table 1.

Table 1. Simulation parameters for EGT-based PC scheme in uplink NOMA

\begin{tabular}{|c|c|}
\hline Simulation Parameter & Parameter values \\
\hline Cells in a network & 2 \\
\hline Cell radius & $389 \mathrm{~m}$ \\
\hline No. of users or population & $\Omega=10,20,30,40,50$ \\
\hline Users locations & Uniformly distributed [15] \\
\hline $\begin{array}{l}\text { Minimum and maximum power levels: } \\
\qquad\left[p_{\min }, p_{\max }\right]\end{array}$ & {$[15 \mathrm{dBm}, 23 \mathrm{dBm}]$} \\
\hline No. of Power levels & $N=6$ \\
\hline Bandwidth $(\mathrm{Hz})$ & $15 \mathrm{Mbps}$ \\
\hline Total sub-carriers & 50 \\
\hline Sub-carrier spacing & $15 \mathrm{KHz}$ \\
\hline Generated service & $285 \mathrm{Kbps}$ \\
\hline $\begin{array}{c}\text { Max. users per sub-carrier } \\
\text { (simultaneously transmitting users) }\end{array}$ & 2 \\
\hline User generates a fixed sized packet & Poisson arrival distribution \\
\hline Sub-carrier allocation & $\begin{array}{l}\text { Global objective maximization } \\
\text { (GOM) [19] }\end{array}$ \\
\hline Propagation model & COST231 Hata model for $\mu$-cell [20] \\
\hline
\end{tabular}




\begin{tabular}{|c|c|}
\hline Modulation for OFDM & BPSK \\
\hline Shadowing distribution & $\begin{array}{c}\text { Log normal shadowing [21], } \\
\mu=0, \sigma^{2}=8 \mathrm{~dB}\end{array}$ \\
\hline Noise power & $-173 \mathrm{dBm} / \mathrm{Hz}$ \\
\hline Stopping criterion $(\varepsilon)$ & 0.005 \\
\hline Maximum energy & $50 \mathrm{~J}$ \\
\hline
\end{tabular}

In Fig. 2, the energy efficiency of our approach in comparison with other PC schemes is presented, where the energy efficiency [17] is defined as the throughput per transmit energy (bps / J) (see Eq. (3)). The generated service request rate is termed as offered load (services/s). As offered load increases, the energy efficiency initially increases due to the increase of the throughput and then decreases due to the excessive increase of the transmit energy. The proposed EGT-based PC outperforms state-of-the-art PC schemes [7-8, 23] because the EGT-based PC algorithm adaptively adjusts the transmit power level of each user in order to maximize the utility function.

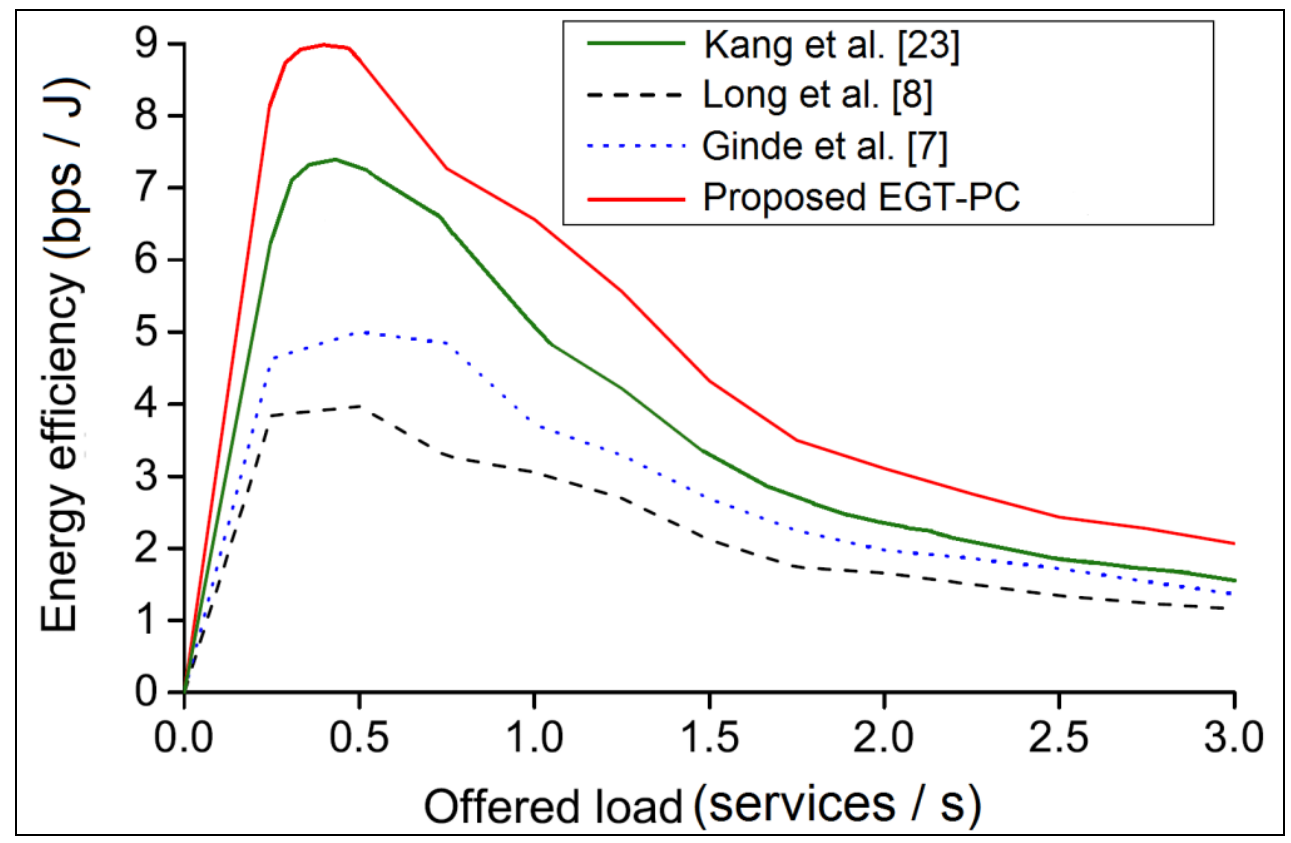

Fig. 2. Energy efficiency of the proposed EGT-based PC approach and comparison with state-of-the-art PC schemes[7-8, 23] 


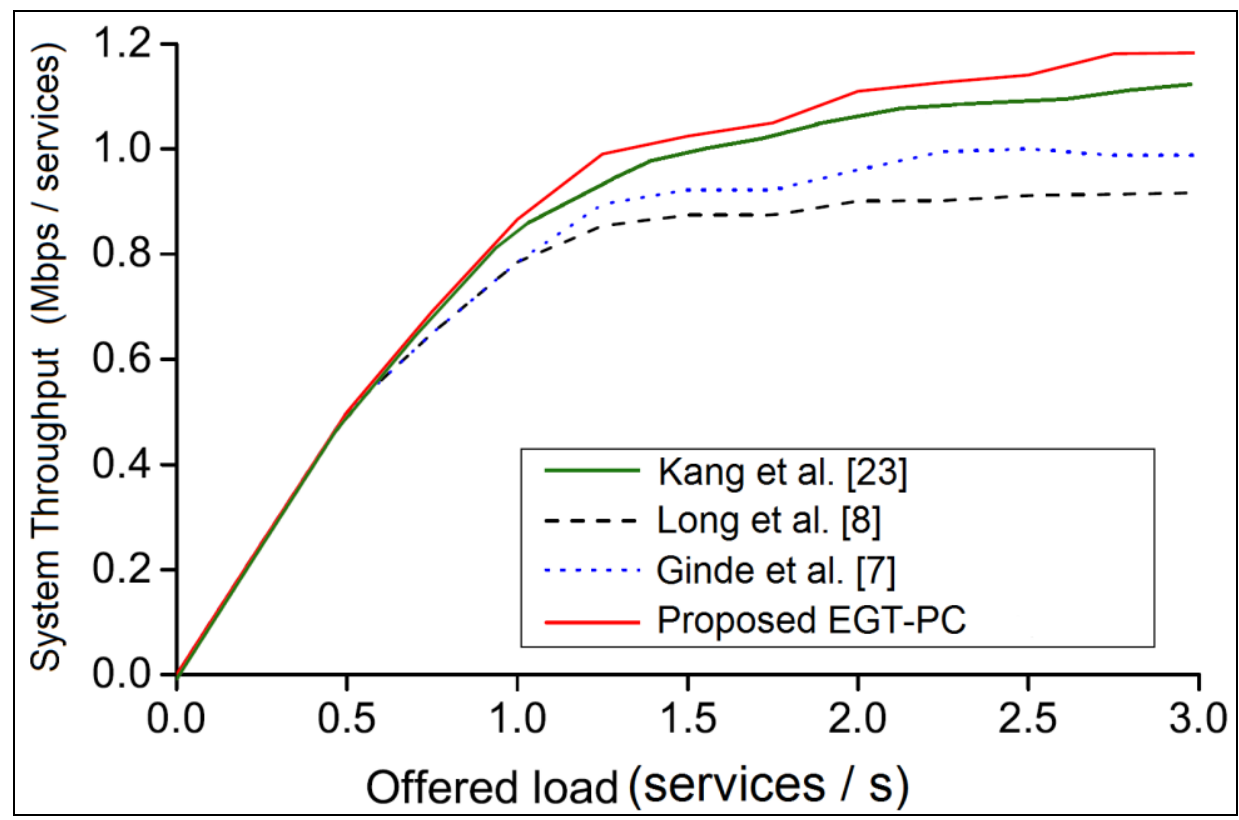

Fig. 3. System throughput of the proposed EGT-based PC approach and comparison with state-of-the-art PC schemes[7-8, 23]

The Fig. 3 presents the throughput per offered load [18] comparison of various PC schemes, where the throughput is defined as the number of accepted service requests in the system. Owing to the dynamics of the strategy adaptation, the EGT-based PC outperforms the traditional PC schemes $[7-8,23]$. The throughput improvement is about $10 \sim 25 \%$ by our approach when the offered load is 1.5 .

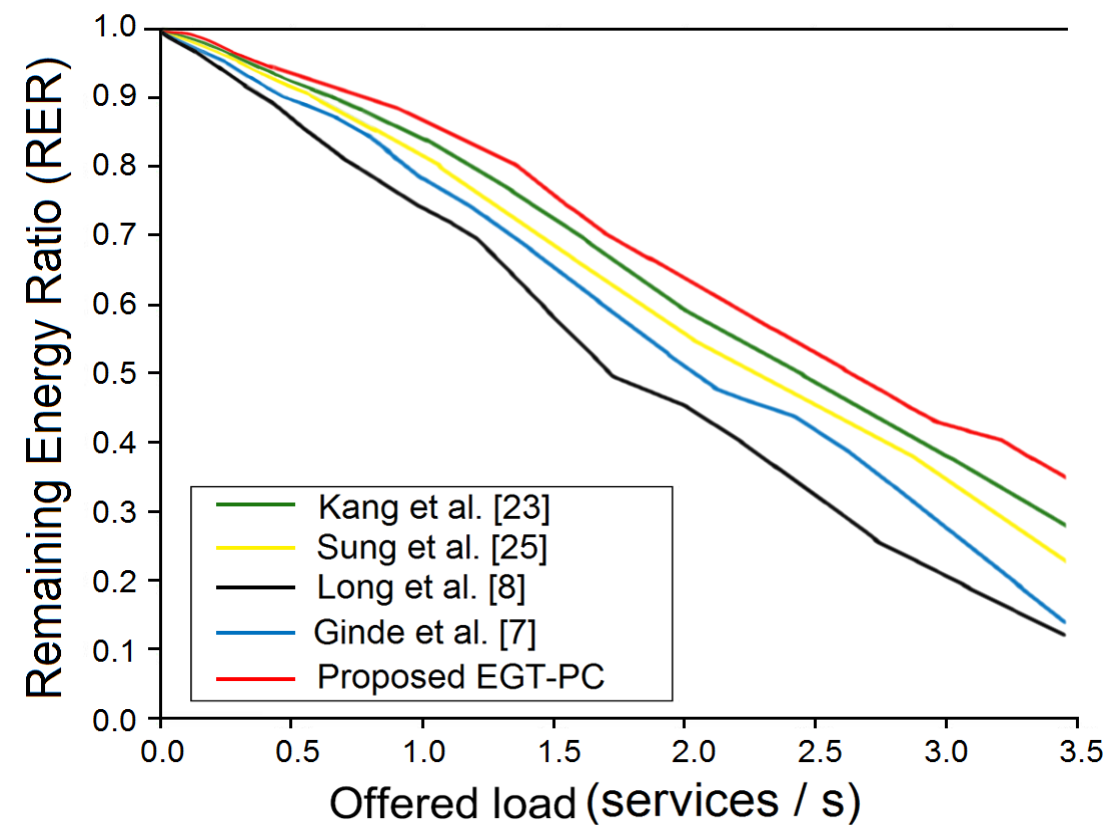

Fig. 4. Comparison of RER for different PC schemes with the proposed EGT-based PC approach 
The proposed scheme is also compared with the state-of-the-art schemes [7-8, 23, 25] in terms of remaining energy ratio (RER) vs. generated service request rate (offered load). The term remaining energy ratio is defined as the ratio of current energy and maximum energy of the system which is 50 Joules. In Fig. 4, we see that the proposed EGT-based PC scheme is more energy efficient when the offered network load increases from 0 3.5. At about $150 \%$ increase in the offered load, the RER of the proposed system is nearly $80 \%$ which is a significantaly better compared to 60 70\% RER achieved by other apporaches.

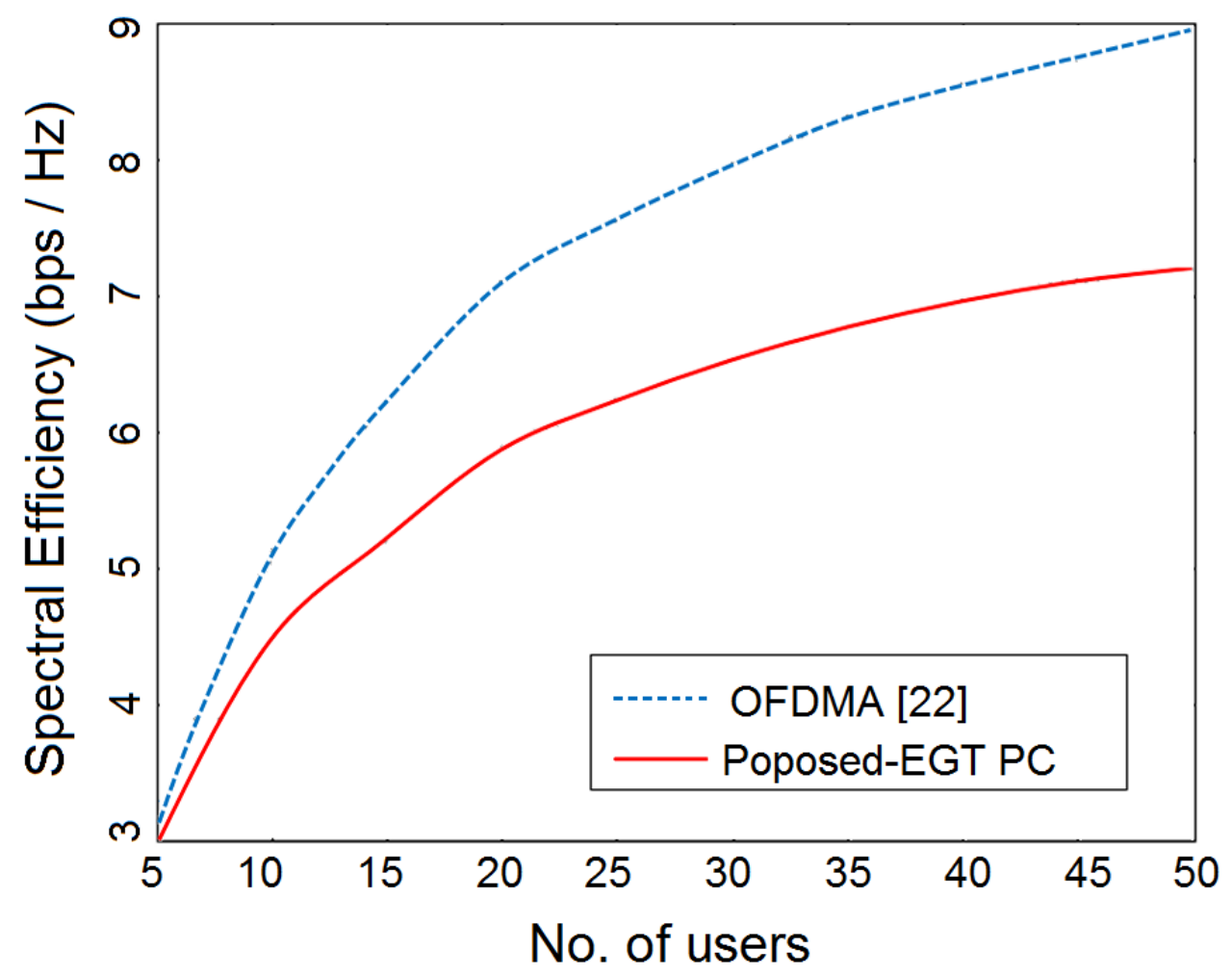

Fig. 5. Comparison of spectral efficiency of the proposed EGT-based PC scheme and OFDMA [22]

Fig. 5 shows the spectral efficiency with respect to the total number of users $(\Omega)$. The spectral efficiency of the proposed EGT-based PC scheme is compared with orthogonal frequency division multiple acces (OFDMA) [22] where proposed approach significantly outperforms in terms of improved spectral efficiency. At $\Omega=50$, the proposed EGT-based PC NOMA scheme achieves spectral efficiency of about $95 \%$, while OFDMA achieves only $80 \%$ of the system upper bound. 


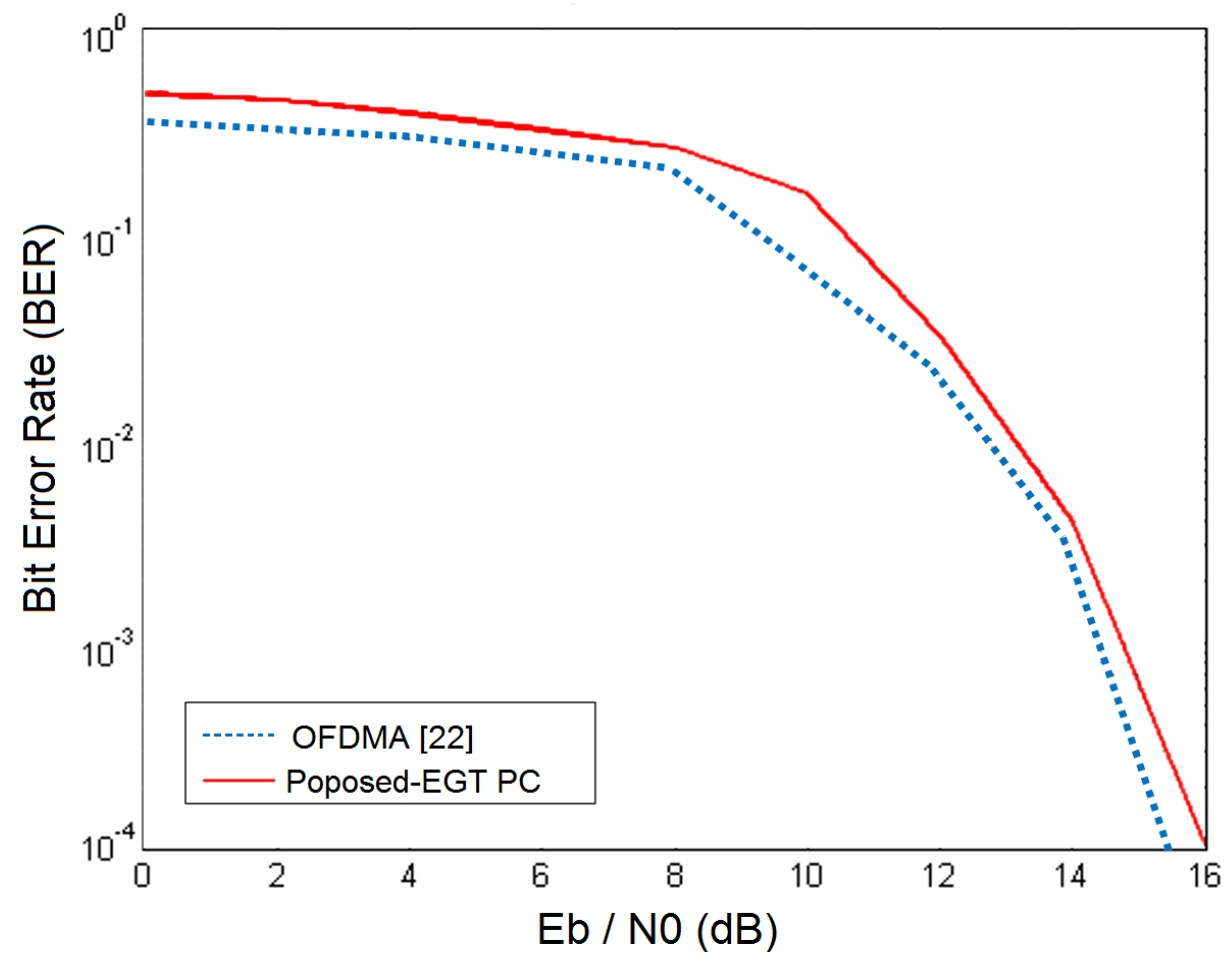

Fig. 6. BER comparison of the proposed EGT-based PC scheme and OFDMA [22]

Fig. 6 shows the bit error rate (BER) vs. $E_{b} / N_{0}$ (energy per bit to noise power spectral density ratio) for the proposed EGT-based PC scheme and OFDMA [22] with the BPSK modulation. The number of sub-carriers are 50 and $15 \mathrm{KHz}$ subcarrier spacing is used. As compared to OFDMA, our proposed approach shows a small BER at the same $E_{b} / N_{0}$. The BER curve shows that there is less interference among the users due to the adaptive power allocation by the proposed EGT-based PC scheme.

\section{Conclusion}

In this study, we presented an evolutionary game theory (EGT) based power control (PC) scheme in uplink NOMA for future 5G networks. In order to serve multiple users simultaneously by power domain multiplexing in the NOMA system, it is important to control the transmit power of the multiple users. We presented how an evolutionary game theoretic model can be adapted for the power-level selection by the individual users in the uplink NOMA system. This paper justified that an EGT-based PC is a good candidate to mitigate the interference between multiple users by adaptive PC. The simulation results show that the EGT-based PC scheme improves the energy efficiency, spectral efficiency, and system throughput compared to other power NOMA schemes and OFDMA.

\section{Acknowledgment}

This work was partly supported by the Sogang University Research Grant of 2012(201210056.01) and MISP(Ministry of Science, ICT \& Future Planning), Korea, under the National Program for Excellence in SW)(2015-0-00910) supervised by the IITP(Institute for Information \& communications Technology Promotion). 


\section{Nomenclature}

\begin{tabular}{|c|c|}
\hline EGT: & Evolutionary Game Theory \\
\hline$P C:$ & Power control \\
\hline NOMA : & Non-orthogonal Multiple Access \\
\hline OFDMA: & Orthogonal Frequency Division Multiple Access \\
\hline$B S:$ & Base station \\
\hline$B \quad:$ & Bandwidth of the system \\
\hline$Y \quad:$ & Received composite signal at the BS \\
\hline$K \quad:$ & No. of simultaneous users (or game players) \\
\hline$N \quad:$ & Total number of strategies or power levels \\
\hline$w:$ & Additive white Gaussian Noise \\
\hline$p_{k}:$ & Transmitted power \\
\hline$s_{k}:$ & Transmitted signal \\
\hline$\Omega \quad:$ & Population or representation of set of players \\
\hline$\nabla \quad:$ & Non-empty set of strategies \\
\hline$\left[p_{\min }, p_{\max }\right]:$ & Minimum and maximum power levels \\
\hline$R_{k}(\boldsymbol{p}):$ & Throughput of player $k$ or data rate \\
\hline$x_{n}:$ & Strategy selection probabilty for selecting $n^{\text {th }}$ power-level \\
\hline$\dot{x}_{n} \quad:$ & Variation of $x_{n}$ \\
\hline$u_{k}$ & Utility function of player $k$ \\
\hline$\lambda\left(x_{n}(t)\right)$ & Control factor to control the speed of convergence \\
\hline$\pi_{n}(t):$ & Payoff of the individual user whose selected strategy is $n$ \\
\hline $\bar{\pi}_{n}(t):$ & Average payoff of the entire population \\
\hline$J(n, m):$ & $\begin{array}{l}\text { Expected payoff of a player using strategy } n \text { and communicating with a player } \\
\text { playing strategy } m\end{array}$ \\
\hline$J(n, X):$ & Expected payoff of a player using strategy $n$ and state of other palyers is $X$ \\
\hline
\end{tabular}

\section{References}

[1] Idate and Umts Forum, "Mobile traffic forecasts 2010-2020 report: A report by the UMTS Forum," in Proc. of UMTS Forum Report 44, January 2011. Article (CrossRef Link)

[2] Linglong Dai, Bichai Wang, Yifei Yuan, Shuangfeng Han, Chih-lin I and Zhaocheng Wang, "Non-orthogonal multiple access for 5G: solutions, challenges, opportunities, and future research trends," IEEE Communications Magazine, vol. 53, no. 9, pp. 74-81, September 2015. Article (CrossRef Link)

[3] Allen B. MacKenzie and Stephen B. Wicker, "Game theory in communications: motivation, explanation, and application to power control," in Proc. of IEEE Global Communications, Houston, USA, pp. 821-826, December 2001. Article (CrossRef Link)

[4] Prabodini Semasinghe, Kun Zhu, and Ekram Hossain, "Distributed resource allocation for self-organizing small cell networks: An evolutionary game approach," in Proc. of IEEE Globecom, Atlanta, USA, pp. 702-707, December 2013. Article (CrossRef Link)

[5] Dusit Niyato and Ekram Hossain, "Dynamics of network selection in heterogeneous wireless networks: An evolutionary game approach," in Proc. of IEEE Trans. Vehicle Technology, vol. 58, no. 4, pp.2008-2017, 2009. Article (CrossRef Link)

[6] Eitan Altman, Rachid ElAzouzi, Yezekeael Hayel, and Hamidou Tembine, "An evolutionary game approach for the design of congestion control protocols in wireless networks," in Proc. of WiOPT, Berlin, Germany, pp.547-552, April 2008. Article (CrossRef Link) 
[7] Samir Ginde, James Neel, and R. Michael Buehrer, "Game theoretic analysis of joint link adaptation and distributed power control in GPRS," in Proc. of IEEE VTC, Orlando, USA, pp.732-736, October 2003. Article (CrossRef Link)

[8] Chengnian Long, Qian Zhang, Bo Li, Huilong Yang, and Xinping Guan, "Non-cooperative power control for wireless ad hoc networks with repeated games," IEEE Journal of Selected Areas in Communications, vol. 25, no. 6, pp.1101-1112, 2007. Article (CrossRef Link)

[9] Alessio Zappone, Stefano Buzzi, and Eduark Jorswieck, "Energy-efficient power control and receiver design in relay-assisted DS/CDMA wireless networks via game theory," IEEE Communications Letters, vol. 15, no. 7, pp.701-703, 2011. Article (CrossRef Link)

[10] Jiashu Li, Jiaming He, Qingbo Zhang, and Shan Huang, "A game theory based WiMAX uplink power control algorithm," in Proc. of 5th International Conference on Wireless Communications, Networking and Mobile Computing, Beijing, China, pp.1-4, 2009.

[11] Emmanouil A. Panaousis, Christos Politis, and George C. Polyzos, "Power control using game theory in a shared open spectrum," in Proc. of Wireless World Research Forum Meeting, 2009.

[12] Tansu Alpcan, Tamer Basar, R. Srikant, and Eitan Altman, "CDMA uplink power control as a noncooperative game," in Proc. of the $40^{\text {th }}$ IEEE Conference on Decision and Control, Orlando, Florida USA, vol. 1, pp. 197-202, December 2001. Article (CrossRef Link)

[13] Andrew Byde, "Applying evolutionary game theory to auction mechanism design," in Proc. of $4^{\text {th }}$ Int. Conference on Electronic Commerce (CEC), ACM, New York, USA, pp.192-193, June 2003. Article (CrossRef Link)

[14] Andrej Kos, and Janez Bester, "Poisson packet traffic generation based on empirical data," Journal of Systems, Cybernetics, and Informatics, vol. 1, no. 5, pp. 80-83, 2003.

[15] Clemens Amstler, and Peter Zinterhof, "Uniform distribution, discrepancy, and reproducing," Kernel Hilbert Spaces, in Journal of Complexity, vol. 17, no. 3, pp. 497-515, 2001. Article (CrossRef Link)

[16] S. M. Riazul Islam, Nurilla Avazov, Octavia A. Dobre, and Kyung Sup Kwak, "Power-domain non-orthogonal multiple access (NOMA) in 5G systems: potentials and challenges," IEEE Communications Surveys \& Tutorials, vol. 19, issue. 2, pp.721-742, October 2016. Article (CrossRef Link)

[17] Fang Fang, Haijun Zhang, Julian Cheng, and Victor C. M. Leung, "Energy efficiency of resource scheduling for non-orthogonal multiple access (NOMA) wireless network," in Proc. of IEEE International Conference on Communications (ICC), Kuala Lumpur, pp. 1-5, 2016. Article (CrossRef Link)

[18] Masahiro Kimura, and Kenichi Higuchi, "System-level throughput of NOMA with SIC in cellular downlink under FTP traffic model," in Proc. of International Symposium on Wireless Communication Systems (ISWCS), Brussels, pp. 1-5, 2015. Article (CrossRef Link)

[19] Pai-Han Huang, Yi Gai, Bhaskar Krishnamachari, and Ashwin Sridharan, "Subcarrier allocation in multiuser OFDM systems: Complexity and approximability and algorithms," in Proc. of IEEE Wireless Communication and Network Conference (WCNC), Sydney, Australia, April 2010. Article (CrossRef Link)

[20] Yuvraj Singh, "Comparison of Okumura, Hata and COST-231 models on the basis of path loss and signal strength," in Intl. Journal of Computer Applications, vol. 59, no. 11, pp. 37-41, December 2012. Article (CrossRef Link)

[21] Anass Benjebbour, Yuya Saito, Yoshihisa Kishiyama, Anxin Li, Atsushi Harada, and Takehiro Nakamura, "Concept and practical considerations of non-orthogonal multiple access (NOMA) for future radio access," in Proc. of Intl. Symposium on Intelligent Signal Processing and Communications Systems (ISPACS), Naha, pp. 770-774, 2013. Article (CrossRef Link)

[22] Yuya Saito, Yoshihisa Kishiyama, Anass Benjebbour, Takehiro Nakamura, Anxin Li, and Kenichi Higuchi, "Non-orthogonal multiple access (NOMA) for cellular future radio access," in Proc. of IEEE $77^{\text {th }}$ Vehicular Technology Conference (VTC Spring), Dresden, pp. 1-5, 2013. Article (CrossRef Link) 
[23] L. P. Qian, Y. Wu, H. Zhou and X. Shen, "Joint Uplink Base Station Association and Power Control for Small-Cell Networks With Non-Orthogonal Multiple Access," IEEE Transactions on Wireless Communications, vol. 16, no. 9, pp. 5567-5582, Sept. 2017. Article (CrossRef Link)

[24] C. W. Sung and Y. Fu, "A Game-Theoretic Analysis of Uplink Power Control for a Non-Orthogonal Multiple Access System with Two Interfering Cells," in Proc. of 2016 IEEE 83rd Vehicular Technology Conference (VTC Spring), Nanjing, 2016, pp. 1-5. Article (CrossRef Link)

[25] K. Kang, Z. Pan, J. Liu and S. Shimamoto, "A game theory based power control algorithm for future MTC NOMA networks," in Proc. of 2017 14th IEEE Annual Consumer Communications \& Networking Conference (CCNC), Las Vegas, NV, 2017, pp. 203-208. Article (CrossRef Link)
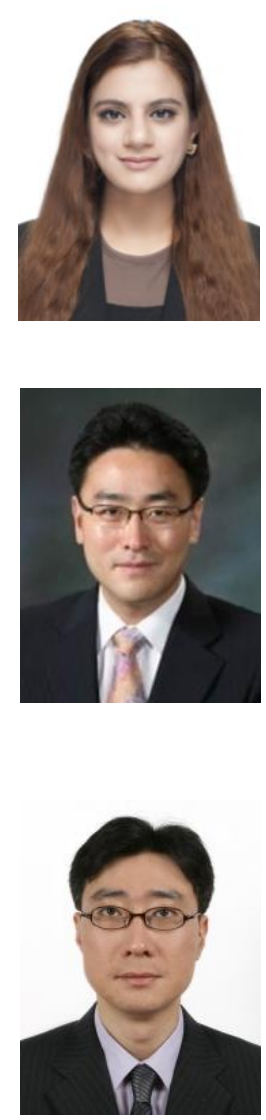

Sidra Riaz received her BS degree in Telecom Engineering from NUCES-FAST, Islamabad, Pakistan, in 2011 and her MS degree in Computer Engineering from Chosun University, South Korea, in 2013. She is currently a Ph.D. candidate in the Department of Computer Science and Engineering, Sogang University, South Korea. Her research interests include wireless transmission, multiple access schemes, and 5G networks. Ms. Sidra Riaz is the recipient of National ICT scholarship award in 2007, Global IT scholarship award in 2011, and Seoul honorary citizenship award in 2017.

Ji-Hwan Kim received the B.E. and M.E. degrees in Computer Science from KAIST (Korea Advanced Institute of Science and Technology) in 1996 and 1998 respectively and Ph.D. degree in Engineering from the University of Cambridge in 2001. From 2001 to 2007, he was a chief research engineer and a senior research engineer in LG Electronics Institute of Technology, where he was engaged in development of speech recognizers for mobile devices. In 2004, he was a visiting scientist in MIT Media Lab. Since 2007, he has been a faculty member in the Department of Computer Science and Engineering, Sogang University. Currently, he is a full professor. His research interests include spoken multimedia content search, speech recognition for embedded systems and dialogue understanding.

Unsang Park received the BS and MS degrees from the Department of Materials Engineering, Hanyang University, South Korea, in 1998 and 2000, respectively. He received the MS and Ph.D. degrees from the Department of Computer Science and Engineering, Michigan State University, in 2004 and 2009, respectively. From 2012, he is an assistant professor in the Department of Computer Science and Engineering at Sogang University. His research interests include pattern recognition, image processing, computer vision, and machine learning. 